Article

\title{
Flexible Fingers Based on Shape Memory Alloy Actuated Modules ${ }^{\dagger}$
}

\author{
Daniela Maffiodo *(1) and Terenziano Raparelli \\ Department of Mechanical and Aerospace Engineering, Politecnico di Torino, 10129 Torino, Italy; \\ terenziano.raparelli@polito.it \\ * Correspondence: daniela.maffiodo@polito.it; Tel.: +39-0110905984 \\ + This paper is an extended version of our paper Maffiodo, D., Raparelli, T.; Comparison among different \\ modular SMA actuated flexible fingers In Proceedings of the The 2nd IFToMM ITALY Conference, Cassino, \\ Italy, 29-30 November 2018.
}

Received: 9 May 2019; Accepted: 7 June 2019; Published: 10 June 2019

check for updates

\begin{abstract}
To meet the needs of present-day robotics, a family of gripping flexible fingers has been designed. Each of them consists of a number of independent and flexible modules that can be assembled in different configurations. Each module consists of a body with a flexible central rod and three longitudinally positioned shape memory alloy (SMA) wires. When heated by the Joule effect, one to two SMA wires shorten, allowing the module to bend. The return to undeformed conditions is achieved in calm air and is guaranteed by the elastic bias force exerted by the central rod. This article presents the basic concept of the module and a simple mathematical model for the design of the device. Experimental tests were carried out on three prototypes with bodies made of different materials. The results of these tests confirm the need to reduce the antagonistic action of the inactive SMA wires and led to the realization of a fourth prototype equipped with an additional SMA wire-driven locking/unlocking device for these wires. The preliminary results of this last prototype are encouraging.
\end{abstract}

Keywords: shape memory alloy; SMA wires; flexible actuator; modular actuator; mathematical model; experimental test

\section{Introduction}

Shape memory alloys (SMAs) are a particular class of metal alloys characterized by two properties: (1) the shape memory effect (SME), the ability to recover a preset geometric shape when subjected to an appropriate temperature change; and (2) superelasticity (SE), the ability to withstand large deformations (up to $10-15 \%$ compared to the initial configuration) without producing permanent effects within a certain range of temperatures. The first discovery of these phenomena dates back to 1932 thanks to the studies of Chang and Read on the AuCd alloy, and in 1938 the transformation was studied in brass (CuZn). However, it was only in 1962 that Buehler discovered the SME in NiTi alloy, and it was from then that actual research on its metallurgy and on its practical applications began. Many other alloys that presented these properties were analyzed, but from the point of view of applications, the most interesting and useful proved to be those of the NiTi group and the $\mathrm{Cu}$ alloys [1].

Since then, SMAs have been used in many fields of engineering: robotics [2-7], biomedical engineering [8-10], and structural engineering [11,12]. The field of innovative robotics seems to be particularly suited to exploiting the advantages that these materials can provide. These advantages are a high power/weight ratio [13], sensing ability, remotability, low driving voltage, simplicity, cleanliness, and silent actuation. Some of these advantages are emphasized when the device decreases in size. On the other hand, they also present disadvantages, such as low energy efficiency, fatigue problems, 
history-dependent characteristics, and low actuation frequency [14]. Much research attempted to overcome these disadvantages, in particular, by implementing different controls in order to obtain a stable and repeatable behavior of their devices [15-17].

Today, industrial automation is entering a new phase, that of the Internet of things (IoT) and the smart factory. Robotics, which is one of the ingredients of this revolution, has the increasing need to find non-conventional solutions. Since the aim is to allow humans and machines to work together without protective barriers, manipulators must also be designed for cooperation.

The end-effector mounted on a manipulator wrist can be a working tool or a gripper. The traditional parallel or angular grippers, with two elements facing each other and operating a parallel or angular movement, are widely used in robotics since they are simple and generally economical $[18,19]$.

Dexterous hands usually have an anthropomorphic shape and the object is grasped by closing mechanical fingers. They are more versatile and are preferred for gripping non-symmetrical objects [20-23]. The actuation system of the grippers can be traditional (electrical, pneumatic) or innovative, like piezo-actuation [24] or SMA actuator-based [16,17,25].

Moreover, flexible actuators are an interesting solution to use as fingers in the gripping hands, in particular for cooperative robots. Different solutions, operated using electric current, hydraulic fluid pressure, or pneumatic pressure [26-28], have been developed, but SMA-operated flexible fingers can also be found in the literature. In general, the solutions implemented with electric motors are less light, smooth, and silent compared to solutions with shape memory materials. Regarding the solutions with hydraulic and pneumatic actuation, they can be used only in environments where the corresponding generation plant is present. Devices with SMA actuation therefore appear to be promising where lightness both for energy system demand and the device itself is required.

As an example, Yang et al. [29] created a flexible device made of three SMA springs embedded off-axially and movably in a silicone rubber rod. A large deformation of the bending actuators are obtained. Drawbacks of their solution are the cooling time, increased by the embedded solution, and friction between the silicone and spring during the motion. Torres-Jara et al. [30] developed a compliant modular actuator based on different arrays of the same simple unit based on a folded sheet of SMA. Linear, rotational and surface actuators are obtained, depending on a different assembly. This is a very interesting solution, both for the design of the object and its performance and for the idea of amplifying the work space by means of the series or parallel composition of a certain number of modules.

The idea of a flexible actuator and that of a modular actuator have been merged together to create the new family of flexible modular actuators presented in this paper. Each module is small, light, and can be variously assembled to allow for the creation of fingers having different shapes and characteristics to satisfy a wide range of needs. Depending on the task, it will be possible to assemble a different number of fingers to constitute various grippers, from the simplest with two fingers for the grasping of simple objects to the anthropomorphic five-finger hand.

This study aims to contribute to this growing area of research by proposing a mathematical model for the design of a flexible actuator, the design and experimental tests performed on four actuators, and the comparison of their different behaviors.

\section{SMA-Actuated Module}

A modular actuator based on shape memory wires is sketched in Figure 1a. The module has a length of $40 \mathrm{~mm}$ and is composed of a central rod (1) with a lower base (2), an upper base (3), and two intermediate disks (4). Three Nitinol SMA wires (5) (diameter $250 \mu \mathrm{m}$ ) are longitudinally placed. One end of each wire is fixed to the lower base and passes through holes in the intermediate disks running parallel to the central rod. This end is now looped through a hole on the upper base and then returns to the lower base where it is fixed. Suitable screws (not shown) placed on the upper base allow the proper tensioning of the SMA wires. Each of the three wires is positioned at $120^{\circ}$ from the others in order to allow the module to bend in any direction when one or more wires are actuated. The module bends when the wire is heated, e.g., by means of the Joule effect, which causes the shortening of the 
wire. When cooled and applying a bias force, the wire is stretched to the original shape and the module comes back to the undeformed shape. This bias force is exerted in this case both by the central rod and by the inactive wires. The wires are not embedded in the structure so as to obtain faster cooling.

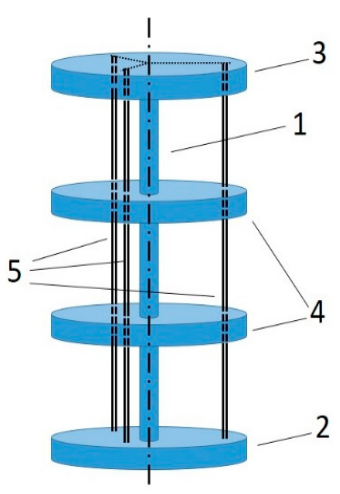

(a)

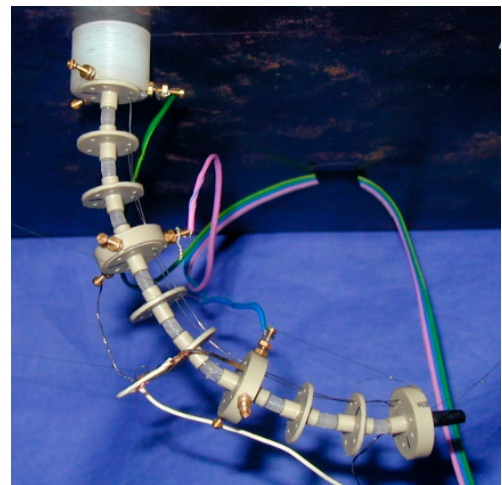

(b)

Figure 1. (a) Sketch of one module: 1) central rod, 2) lower base, 3) upper base, 4) intermediate disks, and 5) three SMA wires @ $120^{\circ}$; (b) Example of a finger composed by three modules in series.

The modules can be joined in order to assemble fingers. This requires a rigid support that constitutes the base on which to arrange the electric power cables, switches, and drives for the control of the structure. As an example, Figure $1 \mathrm{~b}$ shows a finger made of three modules. The heating of one wire will bend the module along one of three main directions (directions 1,2, and 3 in Figure 2). The heating of two SMA wires will bend the module along three secondary directions (directions 1-2, 2-3, and 1-3 in Figure 2).

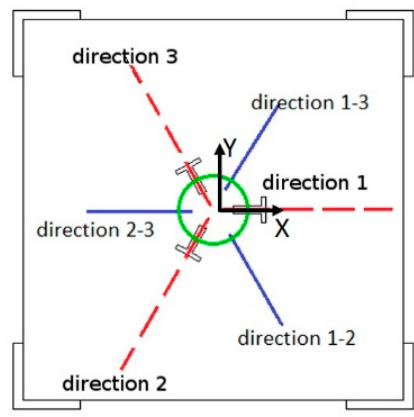

Figure 2. View from above of a module with actuator displacement directions.

The main advantage of this arrangement is that it allows the actuator to move within a volume whose projection on the plane perpendicular to the actuator is $360^{\circ}$; that is, it is possible to carry out all the desired positioning on this plane within the work space of the device.

However, the presence of the other inactive SMA wires, which by their function will be called antagonists, constitutes a physical limit to deformation. Solutions to this limitation are investigated in Section 6.

Figure 3 shows a rendered image of a three-finger gripper and its possible operation. 


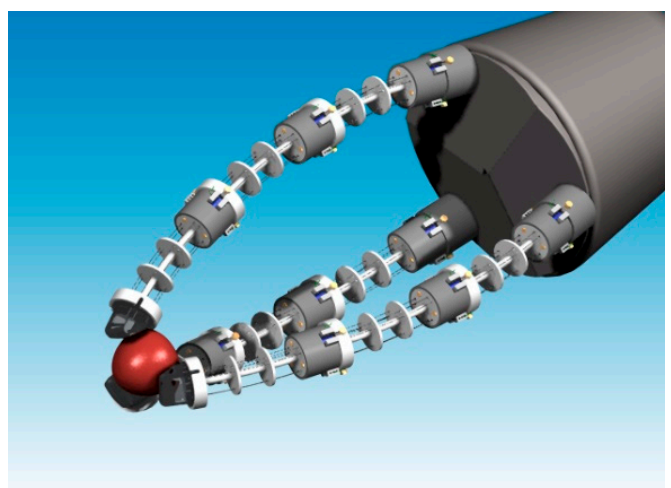

Figure 3. Three-finger gripper.

\section{Mathematical Model}

To evaluate the behavior of a three-module finger, and in particular, the contribution of the antagonistic wires, a numerical analysis was performed. This analysis involves the construction and development of a mathematical model of the system and its implementation and solution. A simplified mathematical model, in which the contribution of the antagonistic wires was neglected, is presented in Maffiodo and Raparelli [31].

This model consists of two parts to distinguish the different behavior in the case of a single SMA wire actuation (motion along the main directions, see Figure 2) and the actuation of two wires (movements along the secondary directions, see Figure 2) where the forces involved and the position of the latter with respect to the center of the device axis varies. Figure 4 shows the sketch of the forces acting on a module in these two different situations.



(a) Case $(A)$

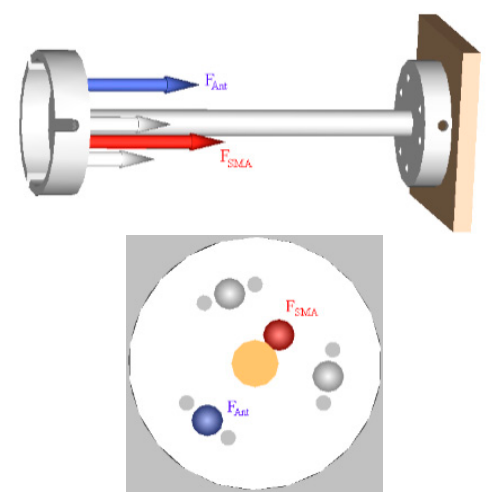

(b) Case (B)

Figure 4. Sketch of the forces acting on a module: (a) lateral view and plant view in the case of one-wire activation (A), (b) lateral view and plant view in the case of two-wire activation (B).

Case A

The displacement along the main directions, as mentioned, is achieved by operating the SMA wires one at a time. In this case, therefore, there will be a force given by the active SMA wire and a double antagonist action determined by the two SMA wires at rest, which is added to the elastic antagonist action determined by the central rod (Figure 4a).

To build a simplified 2D model, the following assumptions were made. With reference to the sketch of Figure $4 \mathrm{a}$, the force of action of the SMA wires (shown in red) is applied to the midpoint of the segment that connects the centers of the two holes in which the SMA wire is running and is directed along the wire itself in the shown direction. The two antagonist forces (shown in gray) are also applied in the midpoints of the segments that connect the centers of the two holes in which the two antagonist SMA wires are running, and are directed along the direction of the wires themselves 
and have the sense represented in the picture. These forces remain parallel to each other during the module deformation; therefore, instead of considering their separate effect, they are replaced by an overall antagonistic force (represented in blue). This antagonistic force has direction and sense equal to the direction of the two antagonistic forces, it has a magnitude that is the sum of the two, and its point of application is located at the midpoint of the segment that connects the two points of application of the two original antagonistic forces. As can be seen in Figure 4a, the actuating force exerted by the SMA wire, the overall antagonist force, and the axis of the actuator are then in the same plane, and therefore the deformed actuator will move along this plane.

Case B

The movement along the secondary directions is obtained by operating the SMA wires two at a time. In this case, therefore, there will be a force due to the two active SMA wires and a single antagonistic action determined by the inactive SMA wire, which is added to the elastic antagonist action determined by the central rod (Figure $4 \mathrm{~b}$ ). To build the 2D model, with reference to the sketch of Figure $4 \mathrm{~b}$, each one of the two SMA forces (shown in gray) is applied at the midpoint of the segment that joins the centers of the two holes along which the SMA wire runs. Similarly to the previous case, these forces remain parallel to each other throughout the deformation; therefore, instead of considering their separate effect, they are replaced by a total SMA force (represented in red). This force has direction and sense equal to the direction of the two SMA forces, it has a magnitude that is the sum of the two, and its point of application is located at the midpoint of the segment that connects the two points of application of the two SMA forces. The antagonist force (represented in blue) is generated by a single wire (the wire at rest); it is applied at the midpoint of the segment that connects the center of the two holes in which the antagonist SMA wire runs, as shown in Figure $4 \mathrm{~b}$.

Also in this case, the total SMA force, the antagonistic force, and the actuator axis are in the same plane; therefore the deformed actuator will move along this plane.

With these assumptions, in both cases the actuator is subjected to forces lying on a plane that will be called the "deformation plane." Therefore, the model is a two-dimensional (2D) model, based on the analysis of the actions during the actuation in the two cases, and it is subsequently extended to the other directions thanks to the symmetry that the actuator presents.

Moreover, in the model, other simplifying hypotheses have been made. The first one concerns the upper head of the actuator, which even after deformation, is supposed to remain perpendicular to the central rod; the second one consists in neglecting the inertia of the system.

Figure 5 is a sketch of the 2D model.

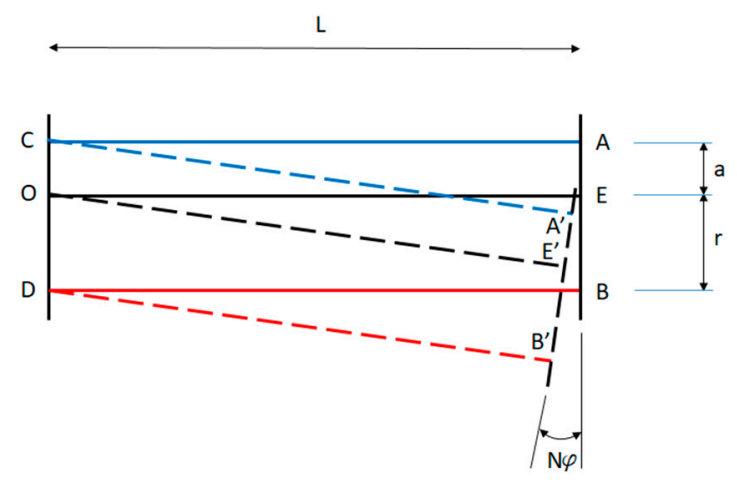

Figure 5. Sketch of the 2D model.

The axis of the actuator is represented by the segment $\mathrm{OE}$, having length $\mathrm{L}$. A generic deformed condition brings the point $\mathrm{E}$ (end-effector) to the position $\mathrm{E}^{\prime}$.

To study this deformation, the actuator is modelled as a set of $\mathrm{N}$ rigid bodies having a length equal to $\mathrm{L} / \mathrm{N}$ each. These elements are connected to each other via torsional springs [31]. For simplicity, 
in the sketch, the deformed configuration starts from point $\mathrm{O}$, the starting point of the first element, and arrives at point $\mathrm{E}^{\prime}$, the final point of the Nth element.

During the actuation, each of these $\mathrm{N}$ elements undergoes a rotation of an angle $\varphi$ with respect to the element that precedes. Therefore, the last element of the rod will be rotated by an angle that is $\mathrm{N} \varphi$ with respect to the horizontal; the upper head, in the final position (segment $\mathrm{A}^{\prime} \mathrm{B}^{\prime}$ ), will form the same angle of value $N \varphi$ with respect to initial position (segment $A B$ ).

The CA segment (drawn in blue) represents the antagonist wire in the rest condition; once the deformation has occurred, this wire changes its configuration, moving to the CA segment and exerting the antagonistic force $\mathrm{F}_{\text {ant }}$.

The DB segment (drawn in red) represents the SMA wire in the rest condition; once the deformation has taken place, this wire also changes its configuration, leading to it coinciding with the DB segment and exerting the $\mathrm{F}_{\mathrm{SMA}}$.

The length $a$ represents the distance between the point of application of the force exerted by the antagonist wire and the axis of the actuator at rest. The length $r$ represents the distance between the point of application of the force exerted by the SMA wire and the axis of the actuator at rest.

It should be noted that the configuration shown in Figure 5 applies both in Case $A$ (main directions) and in Case $B$ (secondary directions). In the latter, parameters $a$ and $r$ and the value of the multiplicative coefficient to be given to the forces involved are different, obtained simply from the different geometry of the system. In Case $A$, the value of the single antagonistic force must be multiplied by two, and in Case $B$, it is necessary to multiply the value of the single SMA force by two.

The device reaches the equilibrium configuration when the sum of the moment of the antagonistic force and of the moment of the elastic reaction of the central rod balances the moment of the SMA force, with respect to the module base (Figure 6). This will occur for a particular angle $\varphi$; in fact, the three moments all depend, obviously in a different way, on this angle. Once the $\varphi$ equilibrium value is obtained, it is possible to determine the elastic deformation of the system, and therefore, its effective working space.

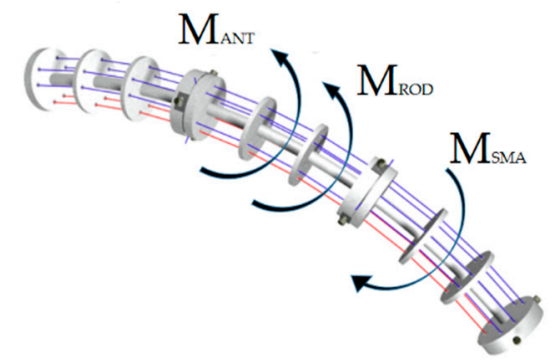

Figure 6. Moments acting on the actuator in the equilibrium condition.

The three considered moments have a different dependence on the deformation angle; therefore, in the following, the procedure that led to the relationships between the forces and the deformation is presented.

\section{Moment of the SMA Force (One Wire)}

In our model, the value of the force exerted by the SMA wire is considered to be constant; in fact, it is assumed that, after a short transient, the wire completes the phase transition and is able to exert the nominal force (value given by the manufacturer). The value of the arm of the force applied by the 
SMA wire depends by the deformation angle $\varphi$. The moment exerted by a SMA wire with respect to the actuator end point is described by the following formula:

$$
M_{S M A}=F_{S M A} \cdot r \cdot \cos \left[a \tan \left(\frac{L \cdot \sum_{i=1}^{N} \sin (i \varphi)}{\left(L \cdot \sum_{i=1}^{N} \cos (i \varphi)\right)-r \cdot \sin (N \varphi)}\right)\right]
$$

Moment of the Antagonistic Force (One Wire)

The moment of an antagonist wire with respect to the actuator base is described by the following formula:

$$
M_{A N T}=F_{A N T} \cdot L_{A N T}=\varepsilon \cdot E A \cdot L_{A N T}
$$

where $A$ is wire section area, $E$ is wire's Young modulus and

$$
\varepsilon=\frac{L_{\text {final }}-L_{\text {start }}}{L_{\text {start }}}
$$

is the longitudinal deformation of the wire.

While the section of the wire and the linear elastic modulus of the material can be considered constant parameters, the value of the longitudinal deformation varies with the angle of deformation because the final length of the wire is a parameter that depends on the angle $\varphi$.

As can be seen, in this case, the deformation angle does not only influence the arm of the force with respect to the final point of the rod but also affects the value of the antagonist force applied by the wire, so the final equations are the following:

$$
\begin{gathered}
F_{A N T}=\frac{E A}{L} \cdot\left[\sqrt{\left(L \cdot \sum_{i=1}^{N} \cos (i \varphi)+a \cdot \sin (N \varphi)\right)^{2}+\left(a-a \cdot \cos (N \varphi)+L \cdot \sum_{i=1}^{N} \sin (i \varphi)\right)^{2}}-L\right] \\
L_{A N T}=a \cdot \cos \left[a \tan \left(\frac{L \cdot \sum_{i=1}^{N} \sin (i \varphi)}{L \cdot \sum_{i=1}^{N} \cos (i \varphi)-r \cdot \sin (N \varphi)}\right)\right]
\end{gathered}
$$

Moment of the Elastic Reaction of the Central Rod

The stiffness of the spring that represents the flexibility of the central rod depends on the material (Young modulus), on the section area, and on the length of the rod itself according to the following relation, so the final moment of the central rod can be described using the following equation:

$$
M_{\text {rod }}=\frac{E_{r} I}{L} N \varphi
$$

where

$E_{r}$ is the Young modulus of the material constituting the rod;

$I$ is the moment of inertia of the rod section;

$L$ is the length of the rod.

\section{Model Results}

By way of example, the results of the model are presented in the present paragraph in the case of a finger consisting of three modules $40 \mathrm{~mm}$ long each, placed in series and having a Polytetrafluoroethylene (PTFE) central rod, with the characteristics summarized in Table 1. 
Table 1. Actuator main data.

\begin{tabular}{cc}
\hline Central rod diameter $(\mathrm{mm})$ & 2.6 \\
Total actuator length $(\mathrm{mm})$ & 120 \\
Young's modulus PTFE $(\mathrm{MPa})$ & 450 \\
SMA wires' positioning radius $(\mathrm{mm})$ & 5 \\
SMA wire diameter $(\mu \mathrm{m})$ & 250 \\
SMA wire shortening $(\%)$ & 5 \\
SMA Young modulus in martensitic phase $(\mathrm{GPa})$ & 28 \\
SMA Young modulus in austenitic phase $(\mathrm{GPa})$ & 75 \\
\hline
\end{tabular}

When the actuator reached the theoretical equilibrium condition, the model gave the results summarized in Table 2.

Table 2. Model results.

\begin{tabular}{ccc}
\hline & Main Direction & Secondary Direction \\
\hline Total SMA moment $(\mathrm{Nm})$ & 0.0424 & 0.0454 \\
Total antagonistic moment $(\mathrm{Nm})$ & 0.0400 & 0.0440 \\
Total central rod moment $(\mathrm{Nm})$ & 0.0024 & 0.0014 \\
Total deformation angle Nf $(\mathrm{rad})$ & 0.028 & 0.017 \\
\hline
\end{tabular}

Figure 7 shows the final deformed configuration of the finger, both along the main directions (dotted line) and the secondary ones (solid line), considering the actuator axis in a rest condition laying along the Z-axis. This graph represents the deformation of the actuator axis on the deformation plane; this plane contained the axis of the actuator, and the forces exerted by the SMA wires and by the antagonist wires.



Figure 7. Axis of central rod of the actuator in equilibrium condition.

Figure 8 shows the projection of the deformation on a plane perpendicular to the actuator axis $(\mathrm{X}-\mathrm{Y})$.

As can be seen, and with reference to Figure 2, the greater deformations occurred along the three main directions, which, starting from the X-axis, were arranged to form three $120^{\circ}$ angles between them. Instead, along the three secondary directions that were also arranged at $120^{\circ}$ between them but $60^{\circ}$ out of phase with the previous ones, the deformations were smaller. This was due to the fact that along the secondary directions, although there were two SMA wires activated at a time and there was only one antagonist wire, the arm of action of the force exerted by the two SMA wires was very small with respect to the arm of the force exerted by the antagonist wire. On the contrary, in the main directions, only one SMA wire was operated, but the arm of the force exerted by the SMA wire was bigger. 




Figure 8. XY projection of the workspace.

\section{Prototypes Designs}

In Table 3, the characteristics of the materials of three different realized prototypes are presented, and Figure 9 shows the prototypes.

Table 3. Prototypes characteristics.

\begin{tabular}{cccc}
\hline & Material & Young's Modulus (MPa) & Melting Temperature $\left({ }^{\circ} \mathbf{C}\right)$ \\
\hline$($ A) & Nylon & 800 & 120 \\
$(B)$ & PTFE & 800 & 250 \\
(C) & PEEK1000/LIM & $4400 / 50$ & 340 \\
\hline
\end{tabular}

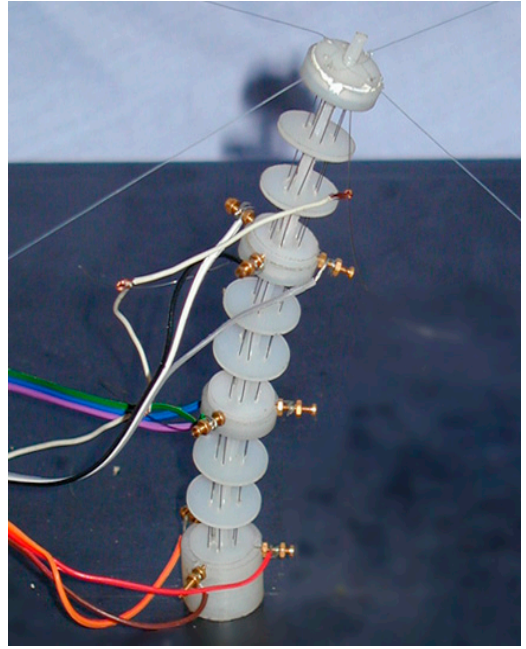

(A)

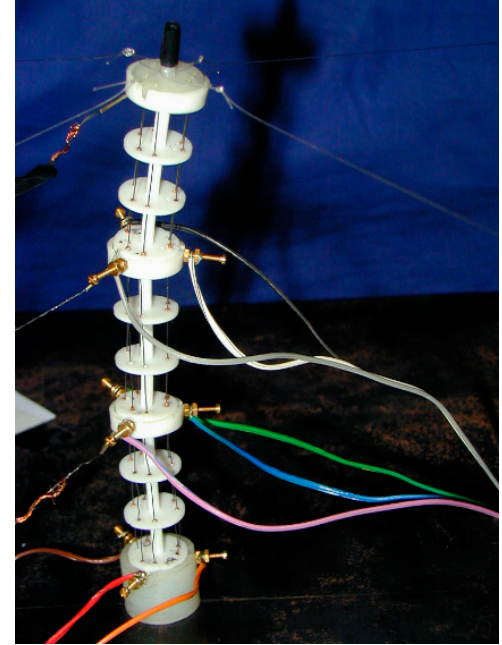

(B)

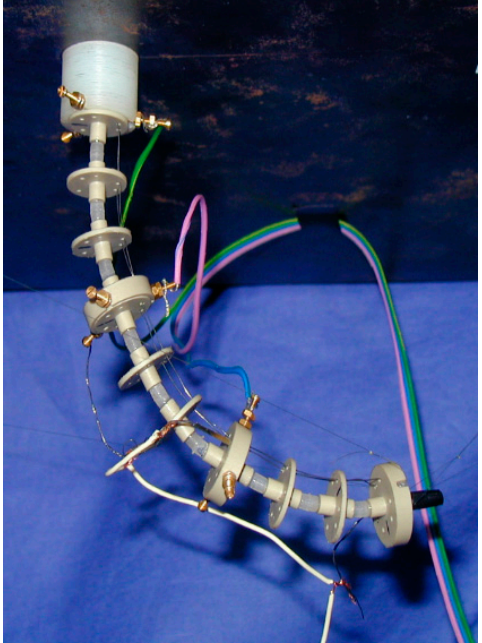

(C)

Figure 9. Prototypes A with nylon central rod (A), B with PTFE central rod (B), and C with LIM central rod and PEEK1000 disks (C).

Due to a Young's modulus of the nylon central rod of about $800 \mathrm{MPa}$, prototype A was characterized by a quite good flexibility. The advantage of this solution is that the material is inexpensive and its machine tooling process is simple, but the disadvantage is that its melting temperature, around $120^{\circ} \mathrm{C}$, 
is close to the temperatures reached by the activated hot wires. Some results of experimental testing on this prototype can be found in Maffiodo and Raparelli [32]. Prototype B, which had a central module made of PTFE, was then built. This material is more expensive, in particular with regard to tool machining. The advantages, however, are a similar Young's modulus, excellent flexibility of the structure, and a decidedly higher melting temperature.

Prototype $C$ was created with the idea of separating the need to have a high melting temperature of the areas in contact with the SMA hot wires and the need to have a high flexibility of the central shaft. Then, the bases and intermediate disks were made with a technopolymer, PEEK1000, with a high Young's modulus and with a high melting temperature, while the flexible elements were made of silicone rubber (LIM) with a low Young's modulus.

\section{Experimental Tests Bench, Procedure, and Results}

The experimental set up, sketched in Figure 10, is composed of a flat square plate with four vertical rods at its edges. The prototype is clamped in the middle of the plate and four low-friction nylon wires are fixed to the actuator upper end. The opposite end of the wire is connected to a load with the aim of tightening the wire itself and to calculate the actuator end position in space using simple algebraic and trigonometric calculations.

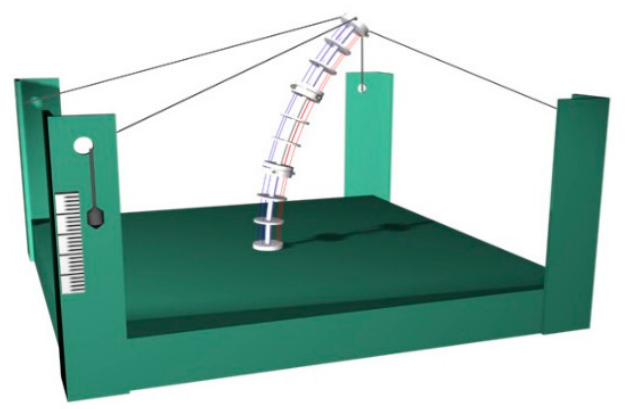

Figure 10. Sketch of the test bench.

The test aimed to evaluate the actuator displacement along the three main directions and the three secondary directions (cf. Figure 2) during heating and cooling sequences. The test was carried out with a step supply current of $1 \mathrm{~A}$ for the heating phase (duration $30 \mathrm{~s}$ ). The activation sequence for each direction was the following: activation of the lower module, activation of the central module, activation of the higher module. Cooling was in still air. Each sequence was repeated at least three times. The power supply was $2.8 \mathrm{~W}$.

The tests performed on prototype A highlighted its stable and repeatable behavior. The 3D workspace, visible in Figure 11, was sufficiently large. The melting temperature close to the temperatures reached by the SMA wire in the hot phase was an important limit of the prototype, which after a relatively low number of cycles had been damaged in these areas. In a first instance, a local insulation can be added, but design changes have to be implemented. 


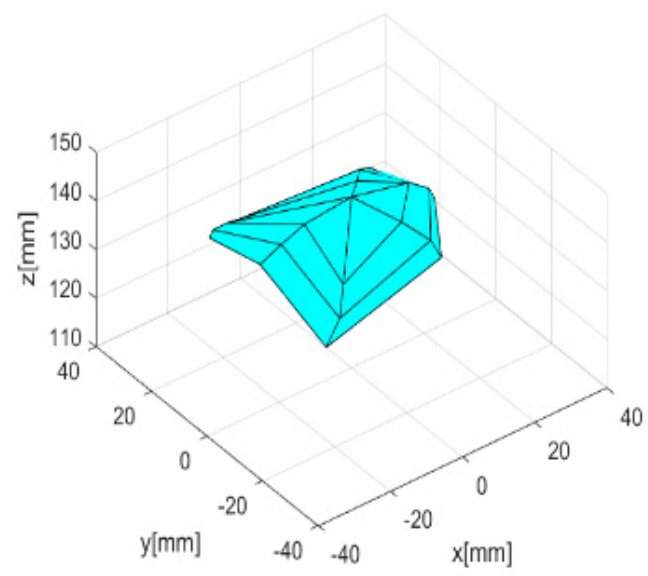

Figure 11. 3D workspace of prototype A.

Prototype B, despite having mechanical characteristics similar to prototype A, had a slightly lower working space. It was observed that this difference can be attributed to a different pretensioning of the antagonist wires in the two prototypes. This observation has led to the need to investigate in more depth the contribution of inactive antagonistic SMA wires, which seemed to significantly limit the deformation of the entire actuator. Figure 12 shows the comparison of the tests obtained on prototype $B$ in the case of the presence of antagonist wires (a), and in the absence of antagonist wires (b). Obviously, this situation, which was artificially generated by disassembling the antagonist wires, is not compatible with the normal use of the device, but is used only for the purpose of investigation. In the result obtained, namely a considerable increase in the working space in the second case, encourages the realization of a new device in which an additional locking/unlocking device allows for the disabling of the antagonist wire when necessary.



(a)

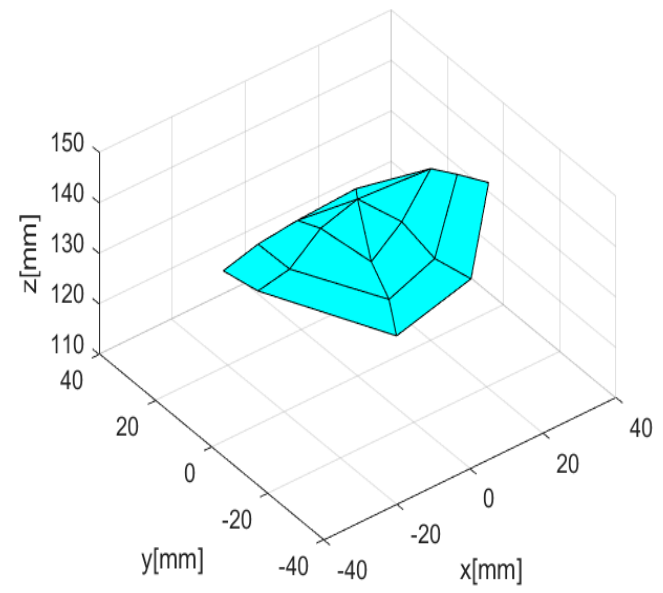

(b)

Figure 12. (a) 3D workspace of prototype B, and (b) 3D workspace of prototype B without antagonistic wires.

Figure 13 shows the comparison of the two different workspaces on an X-Y projection. 


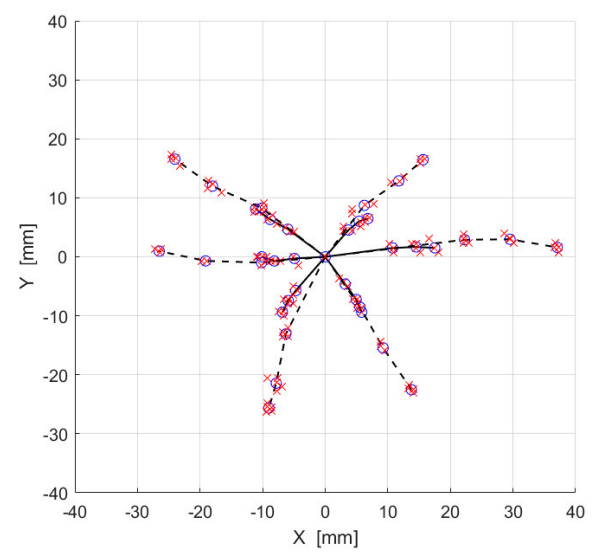

Figure 13. $\mathrm{X}-\mathrm{Y}$ projection of the workspaces for prototype B (cross: experimental, circle: mean values; solid line: with antagonistic wires, dotted line: without antagonistic wires).

Figure 14 shows the results obtained with prototype $C$, which was also tested in the case of the presence of antagonistic wires (a) and in the absence of antagonist wires (b). It can be observed that, also in this case, the 3D workspace, already quite large in case (a), significantly increased in case (b). The device, having good thermomechanical characteristics, was resistant to prolonged use over time. However, silicone rubber was shown to be insufficient to exercise the correct elastic recall for the recovery of undeformed conditions at rest.



(a)



(b)

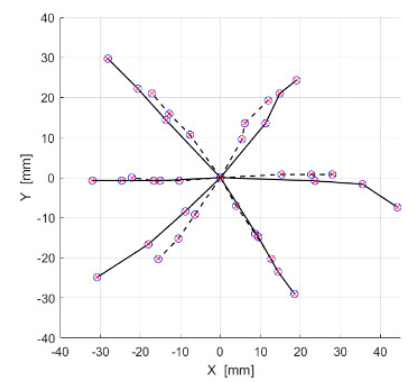

(c)

Figure 14. 3D work space of prototype C: (a) complete; (b) without antagonistic wires; (c) X-Y projection of the workspaces, (cross: experimental, circle: mean values; solid line: with antagonistic wires, dotted line: without antagonistic wires).

\section{New Prototype D}

Based on the results of the experimental tests and the observation that the absence of antagonistic wires would benefit the width of the workspace, a fourth prototype was designed. In this new device, the wires could be blocked for the active phase and released during inactive phases, so as not to work against the active wire. It was, however, necessary that a bias force was present for the return to the undeformed condition of the device. This new device must be as simple and compact as possible, even if the introduction of these new features necessarily involves a greater complexity and an increase in the number of components.

The structure of each module of the new prototype was similar to that of the previous prototypes, except for the addition of the locking/unlocking device housed in a base seat. Figure 15a shows the assembly of the prototype. As can be seen, the addition of a locking/unlocking device has been added below each "traditional" module. In order to facilitate the connection of the wire ends to the cylinder, the distance between the holes for the passage of one wire was modified with respect to the previous solutions $\left(25^{\circ}\right.$ instead of $\left.40^{\circ}\right)$. The materials chosen for the new prototype were the following: PTFE for the module, PEEK100 for the base seat, and PES (Polysulfone) for the cylinders. 


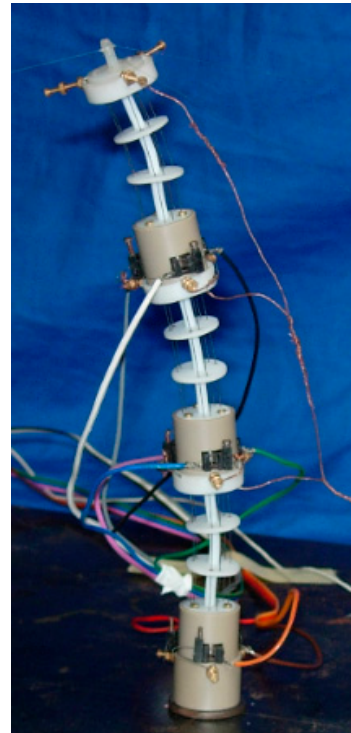

(a)



(b)

Figure 15. (a) New prototype D. (b) Detail of activation/deactivation device on the module base.

Figure 15b shows the detail of the locking/unlocking device. Both ends of each of the three SMA wires were connected to a cylinder (1) located below the lower base. A cylinder (1) was placed inside a gripper (2), which was driven by an additional SMA wire (3). When a current was sent to the additional SMA wire of the gripper, the cylinder was locked and could not move vertically anymore. Subsequently, current was sent to the corresponding SMA wire of the module, which would consequently flex along a main direction. During this phase, the cylinders of the two inactive SMA wires were free to slide vertically because the corresponding grippers were also inactive. Therefore, outside of the friction between the cylinder and gripper, the active wire was not impeded by antagonist wires. When the power supply was interrupted, the clamping action also stopped and the cylinder was free to move again. A similar behavior occurred for the activation along the secondary directions. The bias force for the cylinders and rod was guaranteed by using appropriately sized springs $(\mathrm{k}=2.28 \mathrm{~N} / \mathrm{mm})(4)$.

A set of preliminary tests of the device has been carried out and the results are shown in Figure 16 (continuous black line). The projection in the XY plane of the work space is here compared with the results obtained with prototype $B$, which had a central rod made of the same material. Compared to the solution with antagonistic wires (green solid line), the results of the new prototype were better. Obviously, the presence of sliding friction in the contact between the gripper and cylinder meant that it was not possible to reach the deformation obtained by completely eliminating the antagonist wires from prototype B (green dotted line).

The difference in orientation of the flexion directions of the new prototype with respect to prototype $B$ could be attributed to the sum of two problems related to the experimental procedure: the pretensioning of the SMA wires and the difficulty in orienting the two devices exactly in the same way with respect to the test bench. 


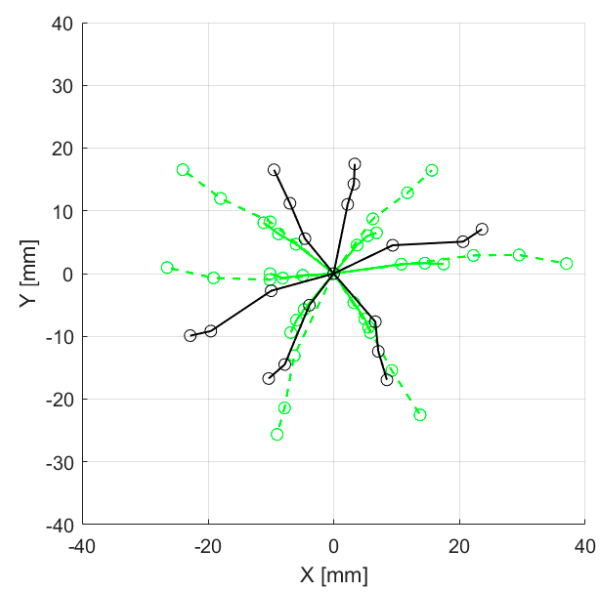

Figure 16. X-Y projection of the workspace with prototype D in black and prototype B in green (solid line: with antagonistic wires, dotted line: without antagonistic wires).

\section{Discussion}

The need to create flexible and versatile gripping hands has led to the creation of a flexible module operated by SMA wires, which can be the basic "brick" with which to assemble many different solutions.

A simple mathematical model was implemented with the aim of theoretically describing the behavior of the device and evaluating its working space.

Based on the results obtained, three devices with the same geometry were developed using three different materials. Experimental tests were conducted on these three prototypes, which allowed the evaluation of the workspace and highlighted some advantages and disadvantages. The working space of prototype A proved to be adequate, but the module was damaged at some points due to the low melting temperature of the nylon. With prototype B, this limit was avoided because the PTFE the central module was made of has a high melting temperature. However, despite a similar nominal elastic module, it showed a reduced working space. Therefore, by accepting an increased difficulty in assembling the module, a solution was conceived, thanks to which large working spaces were obtained and no overheating problems were encountered. This solution (prototype C) involved the use of different materials for the disks and for the central shaft, respectively in PEEK1000 and LIM, but a limitation was introduced by the difficulty in returning to the undeformed condition and a consequent non-repeatability of the tests.

Prototypes B and $C$ were also tested for the evaluation of the influence of the presence of inactive SMA on the work space amplitude, conducted by simply removing these wires and verifying the bending of the central module along the main and secondary directions in the presence of one or two active wires. The results of these tests led to the design of a further prototype. Here, in prototype D, each module had a device for locking/unlocking the SMA wires, governed in turn by an additional SMA wire. Results from the tests on this last prototype are encouraging because its workspace was greater than the workspace of the prototype without a locking/unlocking device.

However, further improvements could be obtained by reducing the sliding friction observed in the new device and by solving some pre-tension and alignment issues. The former could be reduced by taking into consideration other materials for the realization of the gripper/cylinder coupling having a lower friction coefficient, and having greater care for surface roughness. The latter are being resolved through the implementation of appropriate assembly equipment.

Author Contributions: Conceptualization, D.M. and T.R.; methodology, D.M. and T.R.; software, D.M.; validation, D.M.; writing—original draft preparation, D.M.; writing—review and editing, D.M.; supervision, T.R.

Funding: This research received no external funding.

Conflicts of Interest: The authors declare no conflict of interest. 


\section{References}

1. Hodgson, D.E.; Wu, M.H.; Biermann, R.J. Properties and Selection: Nonferrous Alloys and Special-Purpose Materials. In ASM Handbook; ASM International: Metals Park, OH, USA, 1990; Volume 2, pp. 897-902.

2. Raparelli, T.; Beomonte Zobel, P.; Durante, F. Mechanical design of a 3-dof parallel robot actuated by smart wires. In Proceedings of the 2nd European Conference on Mechanism Science, Cassino, Italy, 17-20 September 2008; pp. 271-278. [CrossRef]

3. Raparelli, T.; Zobel, P.B.; Durante, F. A proposed methodology for the development of microgrippers: An application to a silicon device actuated by shape memory alloy wires. Int. J. Mech. Eng. Technol. 2018, 9, 235-249.

4. Lee, K-T.; Lee, G-Y.; Choi, J-O.; Wu, R.; Ahn, S-H. Design and Fabrication of a Smart Flexible Structure using Shape Memory Alloy Wire (SMA). In Proceedings of the 2010 3rd IEEE RAS \& EMBS International Conference on Biomedical Robotics and Biomechatronics, Tokyo, Japan, 26-29 September 2010; pp. 599-603.

5. Wang, Z.; Hang, G.; Li, J.; Wang, Y.; Xiao, K. A micro-robot fish with embedded SMA wire actuated flexible biomimetic fin. Sens. Actuators A 2008, 144, 354-360. [CrossRef]

6. Fukuda, T.; Hosokai, H.; Kikuchi, I. Distributed type of actuators by shape memory alloy and its application to underwater mobile robotic mechanism. In Proceedings of the IEEE International conference on Robotics and Automation, Cincinnati, OH, USA, 13-18 May 1990; pp. 1316-1321. [CrossRef]

7. Maffiodo, D.; Raparelli, T. Three-fingered gripper with flexure hinges actuated by shape memory alloy wires. Int. J. Autom. Technol. 2017, 11, 355-360. [CrossRef]

8. Giataganas, P.; Evangeliou, N.; Koveos, Y.; Kelasidi, E.; Tzes, A. Design and experimental evaluation of an innovative SMA-based tendon-driven redundant endoscopic robotic surgical tool. In Proceedings of the 19th Mediterranean Conference on Control \& Automation, Corfu, Greece, 20-3 June 2011; pp. 1071-1075.

9. Duerig, T.; Pelton, A.; Stöckel, D. An overview of nitinol medical applications. Mater. Sci. Eng. A 1999, 273-275, 149-160. [CrossRef]

10. Petrini, L.; Migliavacca, F. Biomedical Applications of Shape Memory Alloys. J. Metall. 2011, 2011, 501483. [CrossRef]

11. Song, G.; Kelly, B.; Agrawal, B.N. Active position control of a shape memory alloy wire actuated composite beam. Smart Mater. Struct. 2000, 9, 711-716. [CrossRef]

12. Lima, W.M.; Araujo, C.J.D.; Valenzuela, W.A.V.; Rocha Neto, J.S.D. Control of strain in a flexible beam using Ni-Ti-Cu shape memory alloy wire actuators. J. Braz. Soc. Mech. Sci. Eng. 2012, 34, 413-422. [CrossRef]

13. Ikuta, K. Micro/miniature shape memory alloy actuator. In Proceedings of the IEEE International Conference on Robotics and Automation, Cincinnati, OH, USA, 13-18 May 1990; pp. 2156-2161. [CrossRef]

14. Jani, J.M.; Leary, M.; Subic, A.; Gibson, M.A. A review of shape memory alloy research, applications and opportunities. Mater. Des. 2014, 56, 1078-1113. [CrossRef]

15. Shameli, E.; Alasty, A.; Salaarieh, H. Stability analysis and nonlinear control of a miniature shape memory alloy actuatorfor precise applications. Mechatronics 2005, 15, 471-486. [CrossRef]

16. Choi, S.B.; Han, Y.M.; Kim, J.H.; Cheong, C.C. Force tracking control of a flexible gripper featuring shape memory alloy actuators. Mechatronics 2001, 11, 677-690. [CrossRef]

17. Maffiodo, D.; Raparelli, T. Resistance feedback of a shape memory alloy wire. Adv. Intell. Syst. Comput. 2016, 371, 97-104. [CrossRef]

18. Tsugami, Y.; Barbiè, T.; Tadakuma, K.; Nishida, T. Development of Universal Parallel Gripper using Reformed Magnetorheological Fluid. In Proceedings of the 11th Asian Control Conference (ASCC) Gold Coast Convention Centre, Gold Coast, Australia, 17-20 December 2017.

19. Felser, A.; Zieve, P.B.; Ernsdorff, B. Use of Synchronized Parallel Grippers in Fastener Injection Systems. SAE Technical Paper 2015. [CrossRef]

20. Sudsang, A.; Ponce, J. New techniques for computing four-finger force closure grasps of polyhedral objects. In Proceedings of the IEEE International Conference on Robotics and Automation, Nagoya, Japan, 21-27 May 1995.

21. Li, Z.; Hsu, P.; Sastry, S. Grasping and coordinated manipulation by a multifinger robot hand. Intl. J. Robot. Res. 1989, 8, 33-50.

22. Datta, R.; Pradhan, S.; Bhattacharya, B. Analysis and Design Optimization of a Robotic Gripper Using Multiobjective Genetic Algorithm. IEEE Trans. Syst. Man Cybern. Syst. 2016, 46, 16-26. [CrossRef] 
23. Baliga, U.B.; Winston, S.J.; Sandeep, S. Design Optimization of Power Manipulator Gripper for Maximum Grip Force. Int. J. Eng. Res. Technol. 2014, 3, 134-140.

24. Jia, Y.; Zhang, X.; Xu, Q. Design and optimization of a dual-axis PZT actuation gripper. In Proceedings of the IEEE International Conference on Robotics and Biomimetics (ROBIO 2014), Bali, Indonesia, 5-10 December 2014; pp. 321-325. [CrossRef]

25. Modabberifar, M.; Spenko, M. A shape memory alloy-actuated gecko-inspired robotic gripper. Sens. Actuators A Phys. 2018, 276, 76-82. [CrossRef]

26. Schulte, H.F., Jr. The characteristics of the McKibben artificial muscle. In The Application of External Power in Prosthetics and Orthotics; National Academy of Sciences-National Research Council: Washington, DC, USA, 1961; pp. 94-115.

27. Inoue, K. Rubbertuators and applications for robots. In Robotics Research: The 4th International Symposium; Bolles, R., Roth, B., Eds.; MIT Press: Cambridge, MA, USA, 1988; pp. 57-63.

28. Ferraresi, C.; Franco, W.; Quaglia, G. A novel bi-directional deformable fluid actuator. Proc. Inst. Mech. Eng. Part C 2014, 228, 2799-2809. [CrossRef]

29. Yang, K.; Gu, C.L. A compact and flexible actuator based on shape memory alloy springs. J. Mech. Eng. Sci. 2008, 222, 1329-1337. [CrossRef]

30. Torres-Jara, E.; Gilpin, K.; Karges, J.; Wood, R.J.; Russ, D. Compliant Modular Shape Memory Alloy Actuators. IEEE Rob. Autom. Mag. 2010, 17, 78-87. [CrossRef]

31. Maffiodo, D.; Raparelli, T. Design and realization of a flexible finger actuated by shape memory alloy (SMA) wires. Int. J. Appl. Eng. Res. 2017, 12, 15635-15643.

32. Maffiodo, D.; Raparelli, T. Experimental testing of a modular flexible actuator based on SMA wires. Int. J. Appl. Eng. Res. 2018, 13, 1465-1471.

(C) 2019 by the authors. Licensee MDPI, Basel, Switzerland. This article is an open access article distributed under the terms and conditions of the Creative Commons Attribution (CC BY) license (http://creativecommons.org/licenses/by/4.0/). 